\title{
Haemodynamic characteristics of systemic arterial blood supply to the lungs
}

\author{
Fergus Macartney, Philip Deverall, and Olive Scott \\ From the Departments of Paediatric Cardiology and Cardiac Surgery, Killingbeck Hospital, Leeds
}

The haemodynamic characteristics of the systemic arterial blood supply to the lungs were investigated in 9 out of IO patients with absence of the sixth aortic arch, by means of selective catheterization of the major aortopulmonary collateral arteries. Three patients were infants. Total pulmonary resistance was severely raised, and exceeded 9.5 units $\mathrm{m}^{2}$ in all patients while breathing room air, but fell significantly upon breathing Ioo per cent oxygen. The younger patients had a significantly lower pulmonary resistance than the older patients. Important stenoses were shown in the aortopulmonary collateral arteries of all but one patient, either by angiocardiography, pressure measurements, or at necropsy. These stenoses, since they lay distal to any suitable site for surgical anastomosis to a right ventricular conduit, were an important factor contributing to the high total pulmonary resistance. They appear to be present from birth, and probably give rise to the continuous murmurs heard in all these patients.

When the embryonic sixth aortic arch fails to develop or undergoes early involution, pulmonary blood supply is derived entirely from systemic arteries (Manhoff and Howe, 1949; Stuckey, Bowdler, and Reye, 1968). The report of successful surgical correction of such an abnormality (Wallace et al., 1969) prompted us to investigate further the haemodynamic characteristics of pulmonary blood supply in this condition.

\section{Material and methods}

The study consists of ro patients with absence of the sixth aortic arch, as defined by Stuckey et al. (1968). This condition is also known as absence of the pulmonary artery (Manhoff and Howe, 1949; Van Praagh and Van Praagh, 1965), type 4 persistent truncus arteriosus (Collett and Edwards, 1949), and type D pulmonary atresia with a ventricular septal defect (Somerville, 1970). No patient had undergone any previous operation. The diagnosis was established radiographically in each patient by selective injection of contrast medium into the right ventricle and the aorta (frequently both the aortic root and the upper descending aorta). Rapid biplane films were exposed in each patient for a period long enough to see, well opacified, the blood supply to both lung fields, the pulmonary veins, the left atrium, left ventricle, and the aorta on recirculation. A diagnosis of absence of the sixth aortic arch was made if it was not possible to identify either a persistent ductus arteriosus or the main pulmonary arteries proximal to the hila. The detailed

Received 5 June 1972. anatomy of pulmonary blood supply was clarified by selective injection of contrast medium into the large systemic arteries which supplied the lungs (Chesler, Beck, and Schrire, 1970; D. G. Ritter, 1969, personal communication). These arteries were most frequently entered with a Sones coronary arteriography catheter after retrograde catheterization of the aorta from an open axillary arteriotomy. Occasionally it was possible to enter them with a transvenous catheter passed into the aorta across a ventricular septal defect, and if both the above approaches failed, percutaneous femoral artery catheterization was used.

In one patient (Case 5) aged 2 weeks, necropsy showed a fibrous thread running behind the aorta to communicate with intrapulmonary arteries at the right hilum but there was no remnant of the left sixth arch. The vessel from the arch of the aorta supplying the lungs was not a ductus arteriosus, since it was not related to the recurrent laryngeal nerve. In this neonate it was impossible to obtain stable haemodynamic data at the time of catheterization because passage of a catheter down the right subclavian artery in order to enter the ascending aorta resulted in occlusion of the major source of blood supply to the right lung. Haemodynamic data are therefore derived from the remaining 9 patients. Absence of the sixth aortic arch was confirmed in one of these patients (Case 7) at necropsy and in one patient (Case 6) at thoracotomy. In these 9 patients, I7 out of 23 major aortopulmonary collateral arteries were successfully catheterized $(74 \%)$. (In no case was failure to catheterize such an artery seen angiocardiographically to be due to stenosis at its origin.) Definitive haemodynamic studies were carried out at least 20 minutes after any 
angiocardiogram, with the patients routinely premedicated before catheterization ${ }^{1}$. Oxygen saturations were determined from samples drawn from the venae cavae and right atrium, and after inspection of these a right atrial catheter was placed in a position such that it sampled blood at a saturation within 2 per cent of the mean of the saturations in three right atrial sites, the superior vena cava, and the inferior vena cava (a) at the level of the diaphragm and (b) between the entry of hepatic and renal veins. In the patient (Case 9) with an atrial septal defect, the superior vena caval saturation was taken as equal to mixed venous saturation, and the catheter was placed there. The tip of the catheter in the aortopulmonary collateral artery was advanced to a reference point just distal to its first branch, or the most distal point at which surgical anastomosis to a right ventricular conduit might be contemplated. Simultaneous oxygen saturations and pressures were measured in the right atrium, the aortopulmonary collateral artery, and the femoral artery with the patient breathing first room air, and then Ioo per cent oxygen for ro minutes. Further aortopulmonary collateral arteries were then catheterized as far as the reference point already described and the procedure repeated if the oxygen saturation or mean pressure was significantly different. Thus, if the oxygen saturation and pressure were essentially unchanged while breathing room air, the pressure and saturation in one collateral artery during administration of roo per cent oxygen was taken to be representative of those in all the collateral arteries. In only one patient (Case 4) was it possible to obtain a sample of

$1 \mathrm{I} \mathrm{ml} / 20 \mathrm{lb}$ of a mixture containing $25 \mathrm{mg}$ pethidine, $6.25 \mathrm{mg}$ promethazine hydrochloride, and $6.25 \mathrm{mg}$ chlorpromazine $/ \mathrm{ml}$. Maximum adult dose $2.5 \mathrm{ml}$. pulmonary venous blood; in the remainder its oxygen saturation was assumed on the basis of normal ventilation/perfusion ratios in the lung, that is to say 97 per cent breathing room air and roo per cent breathing roo per cent oxygen. Pulmonary venous $\mathrm{Po}_{2}$ was taken to be IOO $\mathrm{mmHg}$ on room air, and $620 \mathrm{mmHg}$ while breathing IOO per cent oxygen. $0.3 \mathrm{ml}$ dissolved oxygen in the plasma was allowed for per $100 \mathrm{ml}$ blood per $100 \mathrm{mmHg}$ $\mathrm{Po}_{2}$ (Slonim, Bell, and Christensen, 1967). Oxygen uptake was determined either directly or from values predicted by LaFarge and Miettinen (1970). Direct measurements were made by the open circuit technique previously described (Kappagoda and Linden, 1972). Pulmonary and systemic flows were calculated by the Fick technique. Pulmonary resistance was defined as the mean of the mean pressures at the reference points in the aortopulmonary collateral arteries $\div$ pulmonary flow index.

\section{Results}

The varieties of pulmonary blood supply encountered are shown in Fig. I. The age of the patients ranged from 2 weeks to 25 years (Table $\mathrm{I}$ ). The sexes were equally represented. One infant (Case 10) would not tolerate 100 per cent oxygen, and in one patient (Case 6) the presence of a $100 \mathrm{mmHg}$ systolic pressure gradient at the orifice of the lower left aortopulmonary collateral artery prevented the meaningful calculation of pulmonary resistance. In all the other patients the mean arterial pressures in the aortopulmonary collateral arteries at the defined reference points were within $10 \mathrm{mmHg}$ of one an-
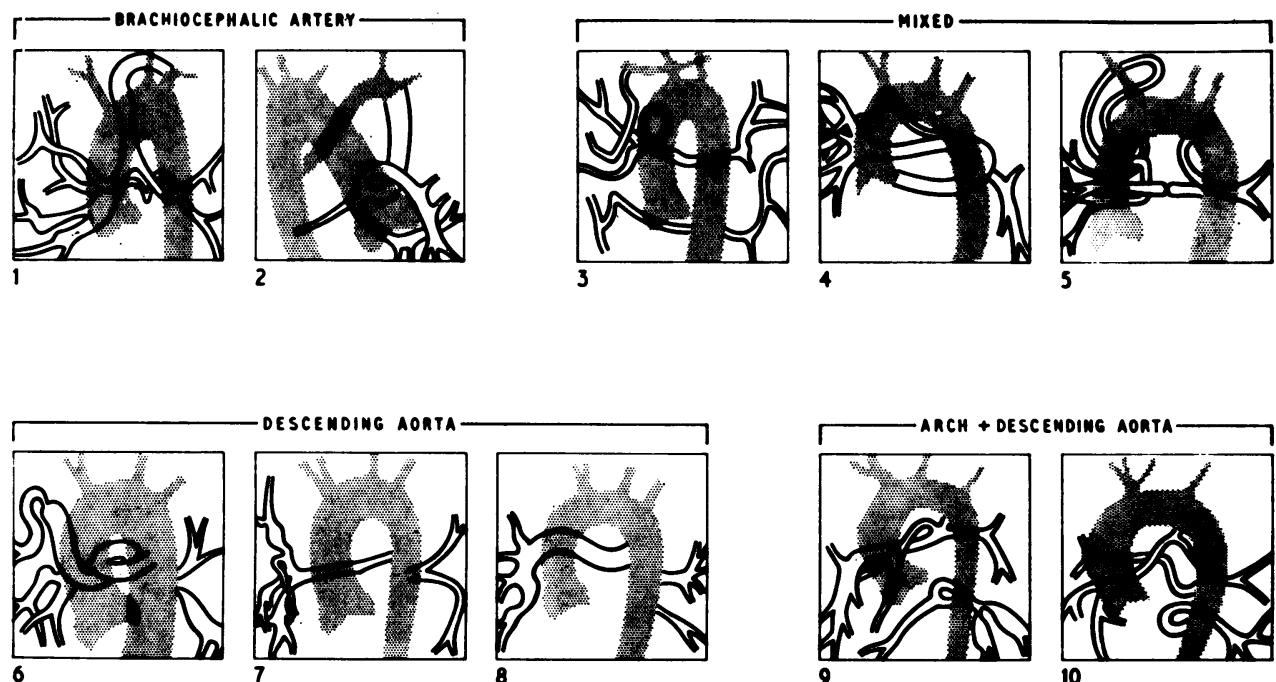

FIG. I Diagrammatic representation of pulmonary blood supply in Io patients with absence of the sixth aortic arch. Major aortopulmonary collaterals are outlined in black; the aorta and its main normal branches are stippled. Patients are identified by the Case No. 


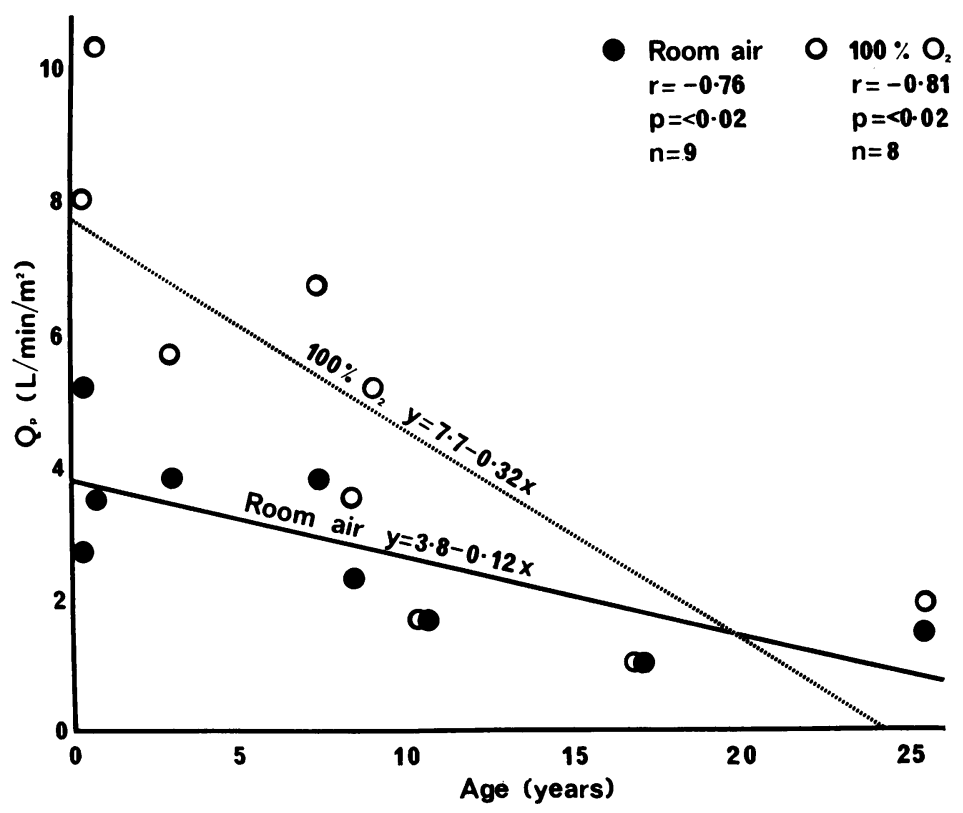

FIG. 2 Relation between age and indexed pulmonary blood flow in 9 patients with absence of the sixth aortic arch, while breathing room air, and 100 per cent oxygen for 10 minutes. One patient would not tolerate 100 per cent oxygen.

TABLE I Details of IO patients with absence of the sixth aortic arch

\begin{tabular}{llllll}
\hline $\begin{array}{l}\text { Case } \\
\text { No. }\end{array}$ & Sex & Age & $H b(\%)$ & $P C V(\%) \begin{array}{l}\text { Systemic } \\
\text { arterial } O_{2} \\
\text { saturation }(\%)\end{array}$ \\
\hline I & F & 10 yr & 113 & 47 & 64 \\
2 & F & 8 yr & 115 & 54 & 72 \\
3 & F & 7 yr & 106 & 40 & 79 \\
4 & M & 8 mth & 83 & 42 & 65 \\
5 & F & 2 wk & 118 & 38 & 52 \\
6 & M & 25 yr & 153 & 64 & 72 \\
7 & F & 16 yr & 125 & 48 & 57 \\
8 & M & 3 yr & 87 & 44 & 63 \\
9 & M & 4 mth & 93 & 40 & 58 \\
I0 & M & 4 mth & 86 & 42 & 76 \\
\hline
\end{tabular}

other in each individual patient, and on average $4.0 \pm 4.0 \mathrm{mmHg}$ lower than the mean femoral arterial pressure. Femoral artery and aortopulmonary collateral artery oxygen saturations were essentially identical. Pulmonary/systemic flow ratios while breathing room air in different patients varied from 0.3 to $I \cdot 3$ (mean 0.69 ). There was no significant correlation between indexed systemic blood flow
(Qs) and the age of the patient. However, indexed pulmonary blood flow $(Q p)$ was significantly $(\mathrm{P}<0.02)$ higher in the younger patients, both while breathing room air and 100 per cent oxygen (Fig. 2). Analysis of covariance showed that, after adjustment for age, mean $Q p$ was significantly $(P<0.05)$ greater while breathing I00 per cent oxygen than while

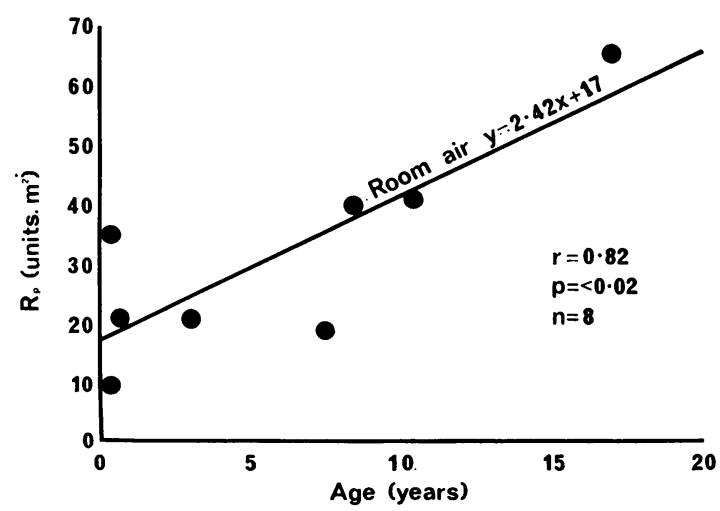

FIG.3 Relation between age and indexed pulmonary resistance in 8 patients with absence of the sixth aortic arch, while breathing room air. 


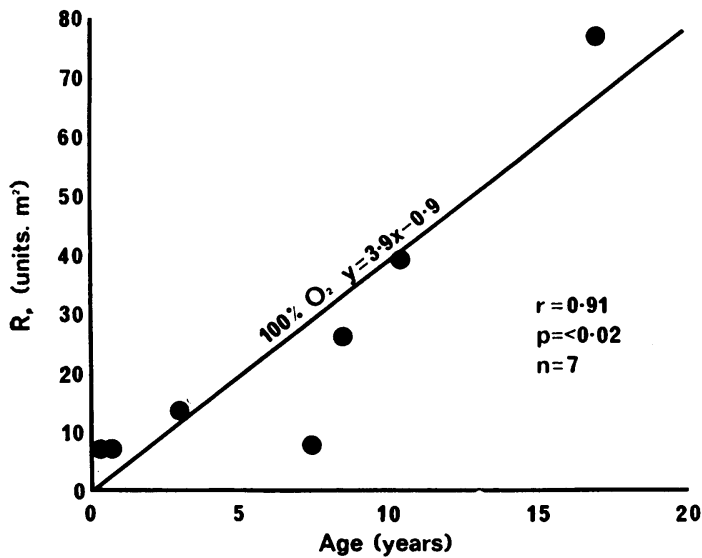

FIG. 4 Relation between age and indexed pulmonary resistance in 7 patients with absence of the sixth aortic arch, after breathing Ioo per cent oxygen for 10 minutes.

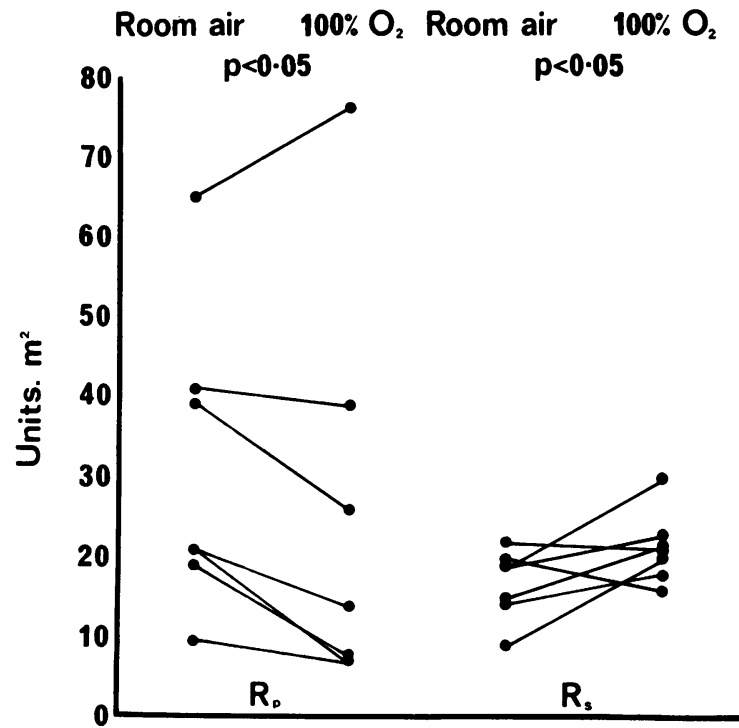

FIG. 5 Effect of breathing 100 per cent oxygen for Io minutes on pulmonary $(R p)$ and systemic (Rs) resistances.

FIG. 6 Selective injection of contrast medium into a major aortopulmonary collateral artery arising from the right subclavian artery in Case 5 (aged 2 weeks) shows a localized severe stenosis of the artery shown at necropsy to lie beneath the parietal pleura of the superior mediastinum, $1.5 \mathrm{~cm}$ proximal to the hilum. 


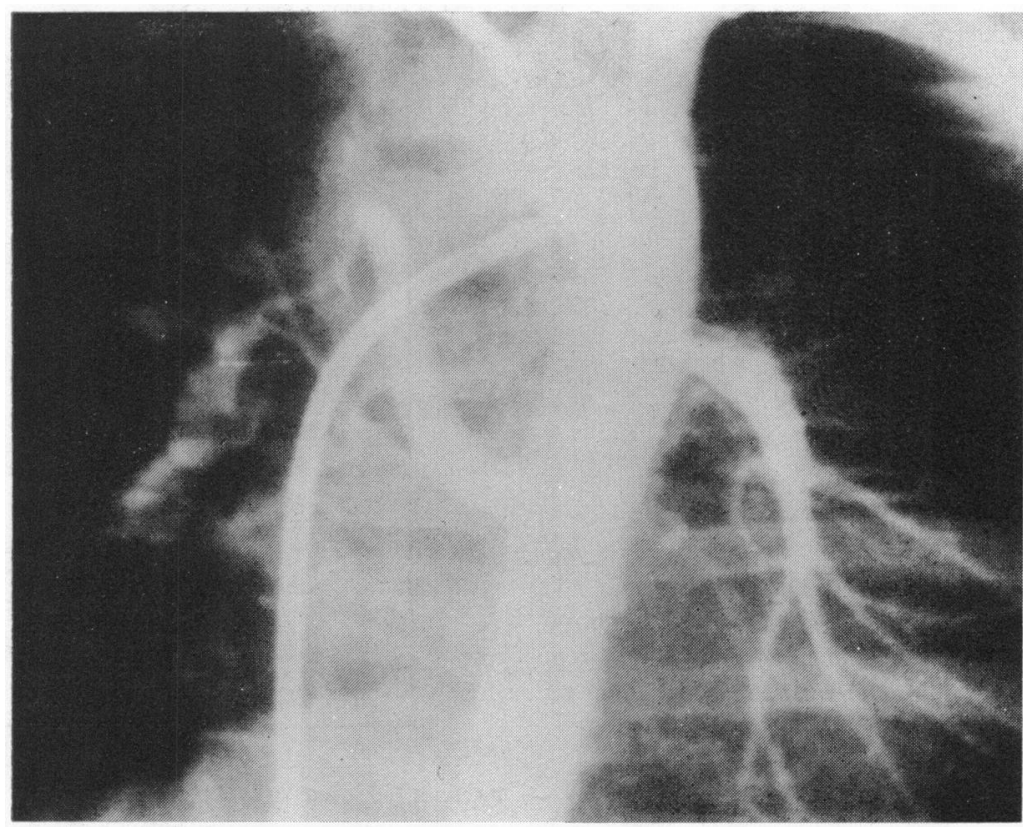

FIG. 7 Selective injection of contrast medium into the upper descending aorta via a catheter introduced into the right axillary artery in Case 4 shows an elongated severe stenosis of the collateral artery supplying part of the right lung. There is a less severe stenosis near the origin of the left-sided collateral artery. A second catheter lies with its tip in the ascending aorta, having traversed a ventricular septal defect.

breathing room air. However, the difference in slopes of the regression lines in Fig. 2 did not achieve statistical significance. Pulmonary resistance $(R p)$ was significantly $(\mathrm{P}<0.02)$ higher in the older patients, both while breathing room air and 100 per cent oxygen (Fig. 3 and 4 ). Over the group as a whole, breathing roo per cent oxygen resulted in a significant fall in pulmonary resistance together with a rise in systemic resistance (Fig. 5). The minimum value of $R p$ encountered while breathing room air was 9.5 units $\mathrm{m}^{2}$ and in only 2 patients did $\mathrm{Rp}$ fall below IO units $\mathrm{m}^{2}$ while breathing 100 per cent oxygen. $\mathrm{Rp} / \mathrm{Rs}$ varied from 0.7 to 3.3 (mean $\mathrm{I} \cdot 92$ ) on room air, and from 0.34 to 4.6 (mean $\mathrm{I} \cdot 36$ ) on roo per cent oxygen.

In all but I patient (Case 3) there was evidence of stenosis of at least one aortopulmonary collateral artery. These had 2 distinct radiological appearances, a very localized constriction (Fig. 6) and a more elongated narrowing (Fig. 7). Evidence of such stenosis was found in 2 patients (Cases 5 and 7 ) at necropsy, and in one of these (Case 5) stenoses were present in all arteries supplying the lungs, sufficiently severe in 2 of them as almost to occlude the lumen. In 3 patients (Cases 7,8 , and 9) systolic pressure gradients of at least $50 \mathrm{mmHg}$ were shown at cardiac catheterization within aortopulmonary collaterals. Blood was sampled with ease distal to the stenoses. In I patient (Case 6) a similar pressure gradient was measured at thoracotomy. In 5 patients, at least one 70 per cent stenosis of a collateral artery was shown angiographically and in all but $I$ patient (Case 3) at least one 50 per cent stenosis was identified. These stenoses were all in the region of the hilum except in I patient (Case 5) (Fig. 6) where the collateral artery illustrated was shown at necropsy to lie just beneath the parietal pleura of the superior mediastinum, posteriorly, with the stenosis $I .5 \mathrm{~cm}$ proximal to the hilum.

The only additional important anomaly apart from the absence of the sixth aortic arch and a high ventricular septal defect, was a secundum atrial septal defect in Case 9.

In 5 patients the systemic arterial oxygen saturation was available from two successive catheterizations (Fig. 8). This had fallen in 3 patients and risen in 2. Despite a maximum interval of 9 years between determinations, the greatest fall in oxygen saturation was only ro per cent.

\section{Discussion}

The normal human pulmonary arterial tree is 
derived from two distinct embryological sources, the embryological pulmonary plexus, which gives rise to the distal pulmonary arteries and veins, and the sixth aortic arches, from which develop the right pulmonary artery, the ductus arteriosus, part of the main pulmonary artery, and possibly some of the left pulmonary artery (Huntington, 1919; Congdon, 1922). Observations support the hypothesis that in pulmonary atresia, the embryological pulmonary plexus has developed normally. The peripheral pulmonary vasculature appears normal both on angiocardiography and after postmortem injection of anomalous collateral arteries. Injection of one substance into the pulmonary arteries and another into anomalous collateral arteries enables identification of the source of blood supply to small pulmonary arteries. Histologically, these are identical regardless of their source of blood supply (Allanby et al., 1950; Dammann and Ferencz, 1956; Ferencz, 1960). When the embryonic pulmonary plexus is first formed during the fourth week of gestation its blood supply is derived mainly from the dorsal aortae, but also from the developing fourth aortic arch and aortic sac (Huntington, 1919; Congdon, 1922; Tobin, 1952). As the sixth aortic arches develop, cranial branches develop from the pulmonary plexus and anastomose to these arches, thus allowing potential communication between the systemic and pulmonary arterial systems. Ultimately the majority of the blood supply comes from the sixth aortic arch. If the sixth aortic arch does not develop or involutes early, the pulmonary plexus continues to derive its only blood supply from systemic arteries. Since none of our patients had been severely cyanosed in infancy and 3 had been shown to have enlarged systemic arteries supplying the lungs at 4 months of age or less (one was 2 weeks old) it is concluded that these vessels have enlarged in utero in response to the absence of the sixth aortic arch. These large systemic arteries have in the past been known as bronchial arteries. The term major aortopulmonary collateral arteries is used in preference here for three reasons: first, because there is some doubt as to whether these vessels have the same embryological origin as bronchial arteries (Boyden, 1970); second, because they do not resemble the more numerous, and much finer systemic collateral arteries (mainly true bronchial arteries) which develop in response to chronic lung disease, or to experimental occlusion of the right or left pulmonary arteries (Liebow, Hales, and Bloomer, 1959), and finally, because not all the anomalous collateral arteries come into relation with the bronchi, as bronchial arteries do (Collister, 1952).

If the development of the embryonic pulmonary plexus is normal, one would predict a vasodilator response of the pulmonary vascular bed in response to breathing 100 per cent oxygen despite its systemic arterial blood supply. Our results showed an overall fall in total pulmonary resistance with 100 per cent oxygen. Since left atrial mean pressure remained unchanged in the one patient in whom it was measured, this fall is presumed to have resulted from dilatation of small pulmonary vessels.

Vasodilatation of pulmonary vessels in response to 100 per cent oxygen cannot be taken as certain evidence that their origin is pulmonary rather than systemic, since there is some evidence that their response to alterations in $\mathrm{Po}_{2}$ is determined by the nature of the perivascular tissue rather than the nature of the vessels themselves (pulmonary or systemic) (Lloyd, 1968, 1970). Nevertheless, the weight of the evidence favours the hypothesis that the only extracardiac anomaly in this condition is

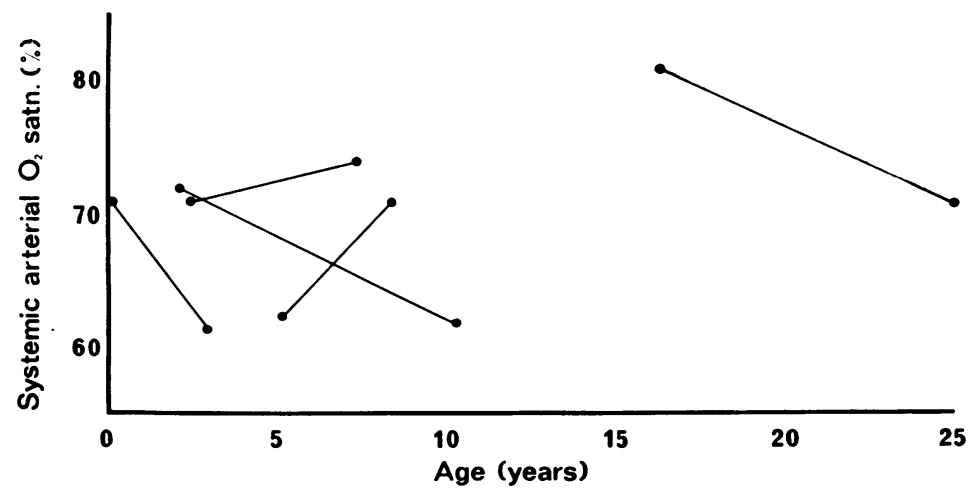

FIG. 8 Systemic arterial oxygen saturations obtained at successive cardiac catheterizations in 5 patients with absence of the sixth aortic arch. 
absence of the embryonic derivatives of the sixth aortic arch. Because normal distal pulmonary arteries are present, 'absence of the pulmonary artery' as a name for this condition (Manhoff and Howe, 1949) is ambiguous, and 'absent sixth aortic arch' (Stuckey et al., 1968) has therefore been adopted. The condition is identical with persistent truncus arteriosus, type 4 of Collett and Edwards (1949), but we do not regard it as a truncus arteriosus communis since this should contain elements of both the fourth and the sixth aortic arches (Van Praagh and Van Praagh, 1965). It is closely related to pulmonary atresia with a sixth aortic arch and a ventricular septal defect both in respect of the appearance of the right ventricular outflow tract and the occurrence of large systemic vessels supplying the lung (Somerville, 1970). However, the extent to which a blind right ventricular outflow tract is present is very variable, and depends upon how much of the distal infundibulum is atretic. It may be impossible to distinguish, even at necropsy, between the right ventricular outflow tracts of a case of absent sixth aortic arch and of true truncus arteriosus communis (R. Van Praagh, 1971, personal communication).

In 4 patients $(40 \%)$ in this series, no blind right ventricular outflow tract was shown by high quality right ventricular angiocardiography. This may not prove that no such outflow tract existed, but emphasizes that, in our view, the diagnosis of this condition is made upon the nature of the pulmonary blood supply, and not upon the degree of infundibular atresia.

The fact that oxygen saturations in femoral artery and major aortopulmonary collateral arteries were identical shows that there is complete mixing of systemic and pulmonary venous blood in this condition. The accuracy of determination of pulmonary blood flow is therefore limited principally by the difficulty of obtaining the pulmonary venous oxygen saturation. Since no patients were in pulmonary oedema, all were ventilating adequately with a normal arterial $\mathrm{PCO}_{2}$, and all appeared to have normal distal pulmonary vessels and central pulmonary veins, it was assumed that pulmonary venous oxygen saturation was normal. Pulmonary resistance (Rp) as calculated, had to be defined in an unusual way as there was no sixth aortic arch present. Since it is a major determinant of operability in all congenital heart lesions associated with pulmonary hypertension, it was defined as an answer to the practical question of what resistance to blood flow would be presented to the right ventricle if the ventricular septal defect were closed and the right ventricle anastomosed to the defined reference points in the arteries providing pulmonary blood supply. In many cases, the number of different aortopulmonary collateral arteries alone would have precluded successful complete correction, but it was felt nevertheless to be profitable to investigate the total resistance to pulmonary blood flow even in these patients as it appeared broadly similar to that offered by a single artery supplying the entire pulmonary blood supply, provided that the mean arterial pressures at the defined reference points in all the major aortopulmonary collateral arteries were equal. In order to estimate the likely error in calculation of $R p$ when these mean pressures were unequal, a mathematical formula was derived (see Appendix) which related this error to the different mean pressures, the relative distribution of pulmonary blood flow, and the number of collateral vessels. The maximum error under the most adverse circumstances in this group of patients appeared to be a Io per cent overestimate of pulmonary resistance, and under average conditions this fell to I per cent. The error is least in those patients with few collaterals and high mean arterial pressures (i.e. the oldest, since mean systemic arterial and mean collateral arterial pressure are approximately equal). Fortunately, it was in those patients that a decision about operability was most critical.

To minimize errors, it is desirable to catheterize every major collateral artery. In fact, 6 such arteries $(26 \%)$ were not catheterized, and none of these appeared angiographically to be a major source of pulmonary blood supply. In only one patient (Case 3) were as many as 2 not catheterized, and this patient had 4 such arteries altogether. Since no angiographic stenosis was shown at the origin of the arteries not catheterized it seems reasonable to assume that the mean pressure therein was at systemic level.

The resistance thus calculated is determined by two factors. These are the resistance offered by the small vessels in the pulmonary vascular bed and the resistance due to stenoses of major aortopulmonary collateral arteries. It was not possible to separate these two determinants except in so far as the fall in resistance on breathing 100 per cent oxygen seemed more likely to be the result of vasodilatation in small vessels than of alterations in major stenoses. The true incidence of stenosis in these arteries is probably greater than that determined in this study because of the difficulty of obtaining intravascular pressures well distal to the hilum, and of interpreting angiocardiograms in which numerous aortopulmonary collateral arteries overlap one another in the region of the hilum. It seems probable that it is arterial stenoses that are responsible for the continuous murmurs audible in all these patients. To what extent these stenoses protect the vascular bed from 
developing obstructive disease is impossible to say. Ferencz (1960) did not find medial hypertrophy or intimal fibrosis in small pulmonary arterioles in her study of the pulmonary vascular bed of patients with tetralogy of Fallot, which included patients with absence of the sixth aortic arch. The size of the arterioles and their relation to arterial stenoses were not specified. However, in these patients, since the stenoses were located distal to a point at which a surgical anastomosis might be made, they limited the chance of successful operation using current techniques as effectively as pulmonary vascular obstructive disease would have done. In none of these patients was operation recommended (even if their age and anatomy had been suitable) because of the high levels of pulmonary resistance. The haemodynamic characteristics of the one patient reported in whom total correction was successful (Wallace $e t$ al., 1969) were much more favourable.

The fact that these arterial stenoses were present in infancy also has important implications for palliative surgery in this condition. Fig. 9 contrasts the situation in the most favourable type of pulmonary atresia with a well-developed sixth aortic arch with the least favourable type of absent sixth aortic arch. Low resistance areas of vascular bed (if they exist) are scattered and probably do not communicate with each other. Banding and shunt procedures are only effective when applied to low resistance vascular beds, and therefore a single palliative procedure of this kind is likely to affect at most a small segment of the pulmonary vascular bed.

Our data suggest that pulmonary resistance starts high and continues to rise in these patients while pulmonary blood flow falls. If this were so one would predict a steady fall in arterial oxygen saturation with increasing age, whereas the limited data from successive catheterizations did not confirm this. It may be therefore that patients with a high pulmonary blood flow came to cardiac catheterization at an earlier age, since they presented with cardiac failure in infancy. The patients studied later in life had no history of cardiac failure. Accurate information as to the variation of pulmonary resistance with age in an individual patient will only come from longitudinal studies in individuals.

Absence of the sixth aortic arch, though it is a severe anomaly, is compatible with remarkably long survival (Manhoff and Howe, 1949; Stuckey et al., 1968). It follows that the natural systemic arterial supply to the lungs is an excellent compensation for the absent sixth aortic arch. Sadly it is often ill suited to be the basis of complete surgical correction.

Grateful thanks are due to Dr. W. Whitaker and Mr. M. I. Ionescu for referring some of the patients for investigation.

\section{Appendix}

Consider a situation in which $\mathrm{N}$ vessels provide a total blood supply $\mathrm{Q} 1$./minute to the lungs. Two of the vessels, $\mathrm{Y}$ and $\mathrm{Z}$, carry proportions $\mathrm{b}$ and a, respectively, of $\mathrm{Q}$. The remainder of the $\mathrm{N}$ vessels carry a flow of $\mathrm{Q}(\mathrm{I}-\mathrm{a}-\mathrm{b}) \mathrm{l}$./minute at a mean pressure $\mathrm{P}$ mmHg. The mean pressures in $\mathrm{Y}$ and $\mathrm{Z}$ are $\mathrm{P}-\mathrm{y}$ and $\mathrm{P}-\mathrm{z} \mathrm{mmHg}$, respectively (i.e. they are stenosed at their origin).

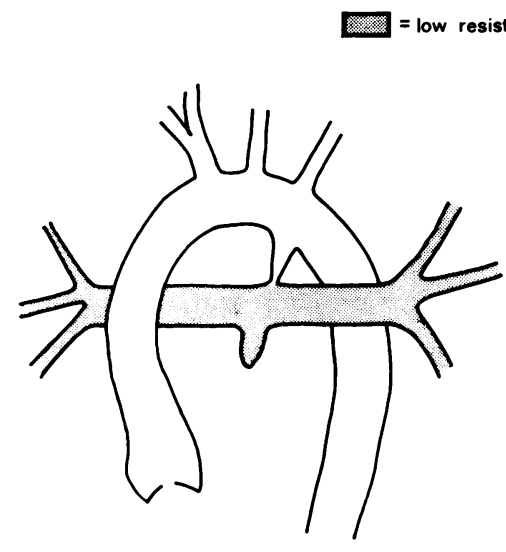

6th aortic arch present

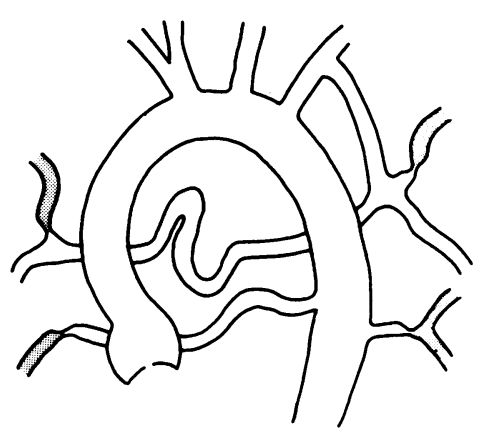

6th aortic arch absent

FIG. 9 Diagrammatic comparison between the haemodynamic state in pulmonary atresia with a ventricular septal defect, with and without a well-developed sixth aortic arch. 
Let $R_{A}$ be the approximate pulmonary resistance as calculated in this study (i.e. mean of mean pressures $\div$ flow)

$$
\mathbf{R}_{\mathbf{A}}=\frac{(\mathbf{P}-\mathbf{z})+(\mathbf{P}-\mathbf{y})+(\mathbf{N}-\mathbf{2}) \mathbf{P}}{\mathrm{NQ}}
$$

The true resistance $R_{T}$ (ignoring frequency dependent components) is given by summing the resistances in individual vessels in parallel.

$$
\frac{I}{R_{T}}=\frac{a Q}{P-z}+\frac{b Q}{P-y}+\frac{Q(I-a-b)}{P}
$$

Solving (2) for $R_{T}$, the error in $R_{A}$ may be determined thus:-

$$
\begin{aligned}
& \text { Error in } \mathbf{R}_{\mathrm{A}}=\frac{\mathbf{R}_{\mathrm{T}}-\mathbf{R}_{\mathrm{A}}}{\mathbf{R}_{\mathbf{T}}}= \\
& z\{N P(a y-a P+b y)-P[y-P-b y+(I-a)(y+z)]-y \\
& \frac{[(b+a-r)(y+z)]\}-P y(y-P-b y+N b P)}{N P(P-z)(P-y)}
\end{aligned}
$$

Inspection of this equation reveals that:-

I) The error is independent of $Q$.

2) When $z=y=0$ the error $=0$, confirming that if all vessels are supplied at the same mean pressure, the approximate resistance $R_{A}$ is accurate regardless of the distribution of blood flow.

In order to analyse the properties of this function further a small digital computer (LINC-8, Digital Equipment Corporation) was used to obtain multiple solutions of the equation for values of $\mathrm{N}$ from 2-5, of $\mathrm{P}$ from 50-90 $\mathrm{mmHg}$, of $y$ and $z$ from o-ro $\mathrm{mmHg}$, and of $\mathrm{a}$ and $b$ from 0 to 0.9 . (Computer printouts are available on request from the authors.)

The results may be summarized thus:-

I) The error may be positive or negative in sign, indicating an underestimate or overestimate, respectively, of $R_{T}$.

2) The error (regardless of sign) increases as $\mathbf{P}$ falls. For this reason values of error quoted below refer to $P=50$ $\mathrm{mmHg}$ (the minimum value encountered in the patients studied) unless stated otherwise.

3) The error (regardless of sign) increases as y (or z) increases if $z$ (or $y)=0$. Values of error are therefore quoted for $y=10, z=10 \mathrm{mmHg}$ (the maximum value allowed in the patients studied) unless stated otherwise. 4) There is increasing overestimation of $R_{T}$ as $N$ increases.

5) When a stenosed vessel carries a small proportion of pulmonary flow, $\mathbf{R}_{\mathrm{T}}$ is underestimated and vice versa.

6) Because of (4) and (5) imposition of a second stenosed vessel on to a system where one vessel only is stenosed usually reduces the error in $\mathbf{R}_{\mathrm{T}}$. This is because if the first stenosed vessel already carries a large proportion of $Q$, the second can only carry a small proportion of $Q$, and therefore tends to reduce the overestimate of $R_{T}$. (e.g. the maximum error for $b=0.8, z=0, y=10$ is - I5.2 per cent when $N=5$.) Raising $z$ from 0 to Io $\mathrm{mmHg}$, i.e. introducing a second stenosis, reduces the error to -12.7 per cent when $a=0.1$. In a system of three vessels with one vessel, $Y$, stenosed, the maximum positive error is $4 \cdot 3$ per cent $(N=3, b=0 \cdot 1, z=0)$.
TABLE $210 \%$ error limits for the calculation of pulmonary resistance as made in these patients

\begin{tabular}{lllll}
\hline$N$ & $P(\mathrm{mmHg})$ & $y(\mathrm{mmHg})$ & $b$ & Error (\%) \\
\hline 3 & 50 & 10 & 0.8 & -12.0 \\
4 & 50 & 7 & 0.9 & -10.6 \\
4 & 50 & 10 & 0.7 & -11.6 \\
4 & 60 & 10 & 0.9 & -13.1 \\
5 & 50 & 10 & 0.6 & -10.4 \\
5 & 60 & 10 & 0.7 & -10.2 \\
5 & 70 & 10 & 0.8 & -10.1 \\
5 & 80 & 10 & 0.9 & -10.0 \\
\hline
\end{tabular}

$\mathrm{N}=$ number of vessels supplying lungs, one of which is perfused at a pressure y $\mathrm{mmHg}$ below the pressure $(\mathrm{P} \mathrm{mmHg}$ ) in the remainder. This vessel carries a proportion $b$ of the total pulmonary blood flow.

The error will be more negative than the value given in the 'Error' column if N, $y$, or $b$ are greater than, or $\mathbf{P}$ is less than, the values given. Positive errors do not reach $10 \%$ under any circumstances in which $\mathrm{P}>50 \mathrm{mmHg}, \mathrm{y}<10 \mathrm{mmHg}, \mathrm{b}>0 . \mathrm{I}$, or $\mathrm{N}<5$ (see Appendix).

Addition of a second stenosis $(N=3, a=0 \cdot I, b=0 . I)$ raises this error to 9.0 per cent. However, this is not much higher than the maximum positive error in a two-vessel system (where only one stenosis is allowable). The error for $N=2, b=0.1$, is 7.8 per cent. So in fact the addition of a second stenosed low flow system only increases the maximum possible positive error by $I \cdot 2$ per cent.

7) Thus, the errors are potentially greatest when only one vessel is stenosed and in this situation are maximal when distribution of flow between the stenosed vessel and the remainder is most uneven. If the stenosed vessel receives $I / N$ th of $Q$ the maximum error is $-I \cdot 3$ per cent. On the other hand, for $N=5, b=0.9$ the error reaches an overall maximum of -17.6 per cent. This requires one stenosed vessel out of five to supply 90 per cent of the pulmonary blood flow, which seems inherently improbable. Table 2 shows the limits within which the error is \pm Io per cent and if it is accepted that a rough idea of flow distribution may be obtained from observing the size of collateral vessels and the volume of lung supplied by them, it seems unlikely that in any patient the error was $> \pm$ ro per cent, particularly as in only one patient was $P<70 \mathrm{mmHg}$. In an average situation $(\mathrm{N}=3, \mathrm{~b}=0.5, \mathrm{y}=4, \mathrm{P}=80)$ the error would be -0.9 per cent.

\section{References}

Allanby, K. D., Brinton, W. D., Campbell, M., and Gardner, F. (1950). Pulmonary atresia and the collateral circulation to the lungs. Guy's Hospital Reports, 99, 1 ro.

Boyden, E. A. (1970). The time lag in the development of bronchial arteries. Anatomical Record, 166, 61 I.

Chesler, E., Beck, W., and Schrire, V. (1970). Selective catheterization of pulmonary or bronchial arteries in the preoperative assessment of pseudotruncus arteriosus and truncus arteriosus Type IV. American fournal of Cardiology, 26, 20.

Collett, R. W., and Edwards, J. E. (1949). Persistent truncus arteriosus: a classification according to anatomic types. Surgical Clinics of North America, 29, 1245. 
Collister, R. M. (1952). Collateral Circulation in Stenosis of the Great Vessels. Uitgeverij Pompe, Leiden.

Congdon, E. D. (I922). Transformation of the aortic-arch system during the development of the human embryo. Contributions to Embryology (Publications of the Carnegie Institution), 14, 47.

Dammann, J. F., Jr., and Ferencz, C. (1956). The significance of the pulmonary vascular bed in congenital heart disease. American Heart fournal, 52, 7.

Ferencz, C. (1960). The pulmonary vascular bed in tetralogy of Fallot. Bulletin of the fohns Hopkins Hospital, 106, 81.

Huntington, G. S. (1919). The morphology of the pulmonary artery in the mammalia. Anatomical Record, 17, 165.

Kappagoda, C. T., and Linden, R. J. (1972). A critical assessment of an open circuit technique for measuring oxygen consumption. Cardiovascular Research, 6, 589.

LaFarge, C. G., and Miettinen, O. S. (1970). The estimation of oxygen consumption. Cardiovascular Research, 4, 23.

Liebow, A. A., Hales, M. R., and Bloomer, W. E. (1959). Relation of bronchial to pulmonary vascular tree. In Pulmonary Circulation, p. 79. Ed. by W. R. Adams and I. Veith. Grune and Stratton, New York.

Lloyd, T. C., Jr. (1968). Hypoxic pulmonary vasoconstriction; role of perivascular tissue. Fournal of Applied Physiology, 25, 560 .

Lloyd, T. C., Jr. (1970). Responses to hypoxia of pulmonary arterial strips in nonaqueous baths. Fournal of Applied Physiology, 28, 566.
Manhoff, L. J., Jr., and Howe, J. S. (1949). Absence of the pulmonary artery: a new classification for pulmonary arteries of anomalous origin. Report of a case of absence of the pulmonary artery and hypertrophied bronchial arteries. Archives of Pathology, 48, 155.

Slonim, N. B., Bell, B. P., and Christensen, S. E. (1967). Cardio-pulmonary Laboratory; Basic Methods and Calculations: A Manual of Cardiopulmonary Technology. Thomas, Springfield, Illinois.

Somerville, J. (1970). Management of pulmonary atresia. British Heart fournal, 32, 641.

Stuckey, D., Bowdler, J. D., and Reye, R. D. K. (1968). Absent sixth aortic arch: a form of pulmonary atresia. British Heart fournal, 30, 258.

Tobin, C. E. (1952). The bronchial arteries and their connections with other vessels in the human lung. Surgery, Gynecology and Obstetrics, 95, 741.

Van Praagh, R., and Van Praagh, S. (1965). The anatomy of common aorticopulmonary trunk (truncus arteriosus communis) and its embryologic implications. American Fournal of Cardiology, 16, 406.

Wallace, R. B., Rastelli, G. C., Ongley, P. A., Titus, J. L., and McGoon, D. C. (1969). Complete repair of truncus arteriosus defects. Fournal of Thoracic and Cardiovascular Surgery, 57, 95 .

Requests for reprints to Dr. F. J. Macartney, Leeds Regional Thoracic Surgical Centre, Killingbeck Hospital, York Road, Leeds LSI4 6UQ. 